\title{
Prevalence and Risk Factors for Opisthorchis viverrini Infections in Upper Northeast Thailand
}

\author{
Kesorn Thaewnongiew ${ }^{1 *}$, Seri Singthong ${ }^{1}$, Saowalux Kutchamart ${ }^{1}$, Sasithorn \\ Tangsawad $^{2}$, Supannee Promthet ${ }^{3}$, Supan Sailugkum ${ }^{4}$, Narong Wongba ${ }^{1}$
}

\begin{abstract}
Opisthorchis viverrini is an ongoing public health problem in Northeast Thailand. Despite continuous efforts for decades by healthcare organizations to overcome this problem, infection rates remain high. To enable related personnel to identify and address the various issues effectively, a cross-sectional study was performed to investigate prevalence and risk factors for opisthorchiasis. The target group was 3,916 Thai residents of Northeast Thailand who were 15 or over. Participants were recruited using the 30 clusters sampling technique. The data were gathered through questionnaires, focus group discussions, in-depth interviews, and stool examinations for parasite eggs (using the Modified Kato Katz method). The data were analyzed using descriptive and inference statistics; in order to ascertain the risk factors and test them using the odds ratio and multiple logistic regressions. The prevalence of opisthorchiasis was $22.7 \%$ (95\% CI: 0.26 to 0.24$)$. The province with the highest prevalence was Nakhorn Phanom (40.9\%; female to male ratio =1:1.2). The age group with the highest prevalence was 40-49 year olds. All age groups had a prevalence $>\mathbf{2 0 \%}$. Four of seven provinces had a prevalence $>\mathbf{2 0} \%$. The factors related to opisthorchiasis were (a) sex, (b) age (especially $>50)$, (c) proximity and duration living near a water body, and (d) eating raw and/or fermented fish. In order to reduce the prevalence of opisthorchiasis, the focus in populations living in upper Northeast Thailand should be changing their eating behaviors as appropriate to their tradition and context.
\end{abstract}

Keywords: Prevalence - Opisthorchis viverrini - risk factors - North East Thailand

Asian Pac J Cancer Prev, 15 (16), 6609-6612

\section{Introduction}

Between 8-10 million people in Asia are infected with the Opisthorchis viverrini (Ov). Sripa et al. (2010) reported that eating raw carp (Cyprinoid fish) infested with Opisthorchis metacercaria the parasite to the gall bladder, resulting in irritation, inflammation and blockages of the biliary system. After decades of chronic inflammation, cholangiocarcinogenesis occurs in subset of infected persons.

Opisthorchis infection among Northeastern directly stems from the habit of eating uncooked infested Cyprinoid fish and fermented fish (rich in nitrosamines a known carcinogen) (Jongsuksuntigul and Imsomboon, 2003).The high incidence and wide-spread prevalence of Opisthorchiasis will persist (Vatanasapt et al., 1990) unless these eating habits are changed and/or the infestations are eliminated (Rangsin et al., 2009).

The respective prevalence of opisthorchiasis in Thailand in 1991, 1996, 2001 and 2009 was 15.2, 11.8, 9.6 and18.6 (Wongsaroj et al., 2009). Rather than curbing consumption of infested fish, rising infection rates suggest the opposite. Interestingly, the variation in prevalence by sub-area was between 19.4 and $41.9 \%$. Sithithaworn et al. (2012) found that in Thailand prevalence was highest up country and in low-lying areas. Wattanayingcharoenchai et al. (2011) confirmed that those at highest risk ate raw fish and uncooked fermented fish and lived near wetlands and canals. Male socialization patterns have men eating raw fish more than females: consequently, an infection rate in males is higher than females (Kaewpitoon et al., 2012a; $2012 b)$. The age group with the highest prevalence was between 20 and 35 , possibly as this group resists changes to patterned socialization (Saengsawang et al., 2012). An important fact was that after eating risky food people could take praziquantel, they return to eating cultural dishes which are likely to lead to a further ov infection. (Wongba et al., 2011). Therefore, the cycle is perpetuated. Previous studied found that the past use of praziquantel associated with OV infection (Saengsawang et al., 2013) and in hamsters infected with ov reported repeated infection and drug treatment can increase the risk of CCA (Charoensuk et al., 2011). Contrast with study of supot kamsa-ard et al. (2013) found that praziquantel treatment and CCA was

${ }^{1}$ Office of Disease Prevention and Control, Khon Kaen Province, ${ }^{3}$ Faculty of Public Health, Khon Kaen University, ${ }^{2}$ Office of Disease Prevention and Control, Saraburi Province, ${ }^{4}$ Vector-born Disease Control, Loei Province, Thailand $*$ For correspondence: Kesthaew@hotmail.com,Thaewnongiew1961@gmail.com 
not statistical significant with a pooled OR of $1.8(95 \% \mathrm{CI}$; 0.81 to 4.16$)$.

The Ministry of Public Health set up a campaign to prevent opisthorchiasis. It did so by providing knowledge to people and cathartic treatment to those diagnosed as having parasite eggs (Jongsuksuntigul and Imsomboon $\mathrm{T}, 2003$ ). A follow-up is, therefore, needed to determine whether opisthorchiasis remains a problem and if so what the current prevalence is by area (having different traditions, environments and local considerations). We set out to(a) investigate (i) the prevalence and (ii) risk factors of those living in upper northeast Thailand and (b) -discover area-specific prevention strategies.

\section{Materials and Methods}

This was a cross-sectional study. The study period was between February and October, 2013. The target provinces were Nakhonphanom, Sakonnakhon, Udonthani, Nongkhai, Bungkan, Nongbualamphu and Loei with a total of 4,568,900 inhabitants. The target group comprised 3,916 people, living in the target area for at least 6 months, and over 15 years of age. All were willing participants.

The subjects were chosen by using 30 Clusters Sampling Technique from 7 provinces which represent the whole population in upper northeast. Modified Kato Katz technique was applied to identify the prevalence of opisthorchiasis. Most of the participants were farmers, living in a variety of geographic locations: plain, plateau and mountains. The Mekong River was not far from some provinces such as Loei, Nongkhai, and Nakhonphanom. Some provinces have natural water resources-i.e., the Songkram canal, Gum canal and Eun canal in Sakonnakhon and Nakhonphanom Provinces and the Nhonghan swamp and some other lakes and canals in Udonthani Province.

The research tools included interviews, focus groups, in-depth interviews and stool testing (Modified Kato Katz method). The research team started the survey after the research protocols had been considered and approved by the Human Research Ethics Committee at Khon Kaen Hospital (21/10/2013). The descriptive statistics and analytical statistics were introduced to explain the relationship of involved factors related to Opisthrochiasis using the chi-square test, Odd ratio, and Multiple Logistic Regression Analysis.

\section{Results}

Stool testing was done on 3,916 persons in the target group. The prevalence of opisthorchiasis was $22.7 \%$ (95\%CI, 0.26 to 0.24$)$ : the highest prevalence was in Nakhonphanom (40.9\%). In four of the seven provinces, the prevalence was $>20 \%$ (Figure 1 ).

The top 10 villages among the 210 surveyed, which had the highest prevalence of opisthochiasis are presented in Table 1. Ankam village, Tao Ngoi had the highest at $80 \%$.

The highest prevalence was among those between 40 and 49 years of age, representing $49.6 \%$ and each age group had a prevalence> $20 \%$ (Figure 2).

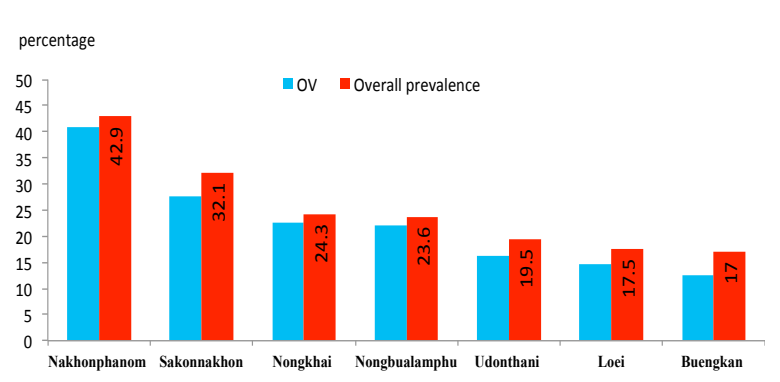

Figure 1. Prevalence of Opisthorchiasis by Area

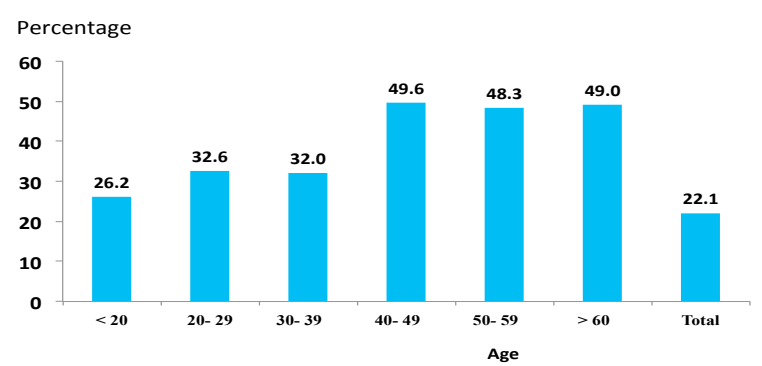

Figure 2. Prevalence of Ophisthochiasis by Age Group

Table 1. Top 10 Villages with the Highest Prevalence of Opisthochiasis in Upper Isaan

\begin{tabular}{|c|c|c|c|c|c|}
\hline Village & District & Province & $\begin{array}{l}\text { No. } \\
\text { stool }\end{array}$ & $\begin{array}{c}\text { Overall } \\
(\%)\end{array}$ & $\begin{array}{l}\text { OV } \\
(\%)\end{array}$ \\
\hline Ankam & Tao Ngoi & Sakonnakhon & 25 & 84 & 80 \\
\hline Kongswang & Banphang & Nakhonphanom & 23 & 78.3 & 78.3 \\
\hline Donpang & Banphang & Nakhonphanom & 12 & 75 & 75 \\
\hline Thai sammakee & Muang & Nakhonphanom & 18 & 72.2 & 72.2 \\
\hline Maijampa & Tart Phanom & Nakhonphanom & 29 & 72.4 & 68.9 \\
\hline Nhongbuakam & Faorai & Nongkhai & 16 & 62.5 & 62.5 \\
\hline Hamhakngaew & Ponsawan & Nakhonphanom & 23 & 65.2 & 60.9 \\
\hline Nonmunpla & Faorai & Nongkhai & 17 & 58.8 & 58.8 \\
\hline Huaihai & Ponsawan & Nakhonphanom & 29 & 62.1 & 58.6 \\
\hline Pukratae & Srisongkam & Nakhonphanom & 29 & 58.6 & 58.6 \\
\hline
\end{tabular}

Table 2. Prevalence of Ophisthochiasis in Upper Isaan

\begin{tabular}{crc}
\hline Prevalence of ophisthochiasis $(\%)$ & $\mathrm{n}=210$ & $\%$ \\
\hline $61-80$ & 7 & 3.3 \\
$40-60$ & 34 & 16.2 \\
$21-40$ & 55 & 26.2 \\
$1-19$ & 93 & 44.3 \\
0 & 21 & 10 \\
Total & 210 & 100 \\
\hline
\end{tabular}

Prevalence can be classified as level of infection in each area. The area where has percentage of prevalence between $1-20 \%$ covers $44.3 \%$. The minor position is prevalence's percentage between $21-40 \%$ which covers $26.2 \%$. However, the area with has $0 \%$ of prevalence covers $10 \%$ (Table 2 ).

\section{Factors related to infection with ophisthochis}

After doing the univariate analysis of ophisthochis infection, the factors related to infection were (a) sex $1.5(1.29$ to 1.73 ), (b) age over 402.1 (1.56 to 2.84), (c) career 1.6 (1.3. to 1.55$)$, (d) water resource habitat $1.6(1.38$ to 1.90$)$, (e) time lived in that area $1.5(1.25$ to 1.67), (f) alcohol consumption 1.4 (1.16 to 1.56$)$, (g) having ophisthochis infection 2.1 (1.59 to 2.73 ), (h) eating 
Table 3. Relationship among Factors in Opisthorchis Infection when Variables Controlled

\begin{tabular}{|c|c|c|c|c|}
\hline Factors & $\begin{array}{c}\text { Total } \\
\text { number }\end{array}$ & $\begin{array}{c}\text { Infected } \\
\%\end{array}$ & $\begin{array}{c}\text { Crude OR } \\
(95 \% \mathrm{CI})\end{array}$ & $\begin{array}{c}\text { Adjusted } \\
\text { OR(95\% CI })\end{array}$ \\
\hline \multicolumn{5}{|l|}{ Sex } \\
\hline Female & 2,134 & 21.6 & ref & ref \\
\hline Male & 1,782 & 29.2 & $1.5(1.29-1.73)$ & $1.2(1.01-1.44)$ \\
\hline \multicolumn{5}{|c|}{ Age (years) } \\
\hline$<20$ & 455 & 14.3 & ref & ref \\
\hline $20-29$ & 500 & 18.8 & $1.3(0.93-1.84)$ & $1.2(0.80-1.67)$ \\
\hline $30-39$ & 665 & 22.6 & $1.8(1.34-2.51)$ & $1.4(0.96-1.95)$ \\
\hline $40-49$ & 881 & 26.2 & $2.1(1.56-2.84)$ & $1.4(0.97-2.00)$ \\
\hline $50-59$ & 750 & 32.1 & $2.5(1.84-3.38)$ & $1.6(1.09-2.28)$ \\
\hline$>60$ & 665 & 29.9 & $2.5(1.82-3.36)$ & $1.5(1.05-2.27)$ \\
\hline \multicolumn{5}{|c|}{ Water Resource Habitat } \\
\hline No & 1,324 & 19.6 & ref & ref \\
\hline Yes & 2,592 & 27.8 & $1.6(1.35-1.86)$ & $1.5(1.26-1.73)$ \\
\hline \multicolumn{5}{|c|}{ Having used to take medicine for Opisthorchis } \\
\hline No & 3,505 & 25.4 & ref & ref \\
\hline Yes & 411 & 21.4 & $0.79(0.62-1.02)$ & $1.4(1.06-1.80)$ \\
\hline \multicolumn{5}{|c|}{ Having Opisthorchis infection record } \\
\hline No & 3,682 & 24.1 & ref & ref \\
\hline Yes & 234 & 40.2 & $2.08(1.59-2.73)$ & $1.8(1.31-2.39)$ \\
\hline \multicolumn{5}{|c|}{ Eating raw fish } \\
\hline No & 1,834 & 15.2 & ref & ref \\
\hline Yes & 2,082 & 33.7 & $2.86(2.44-3.34)$ & $2.2(1.78-2.59)$ \\
\hline \multicolumn{5}{|c|}{ Eating fermented fish } \\
\hline No & 520 & 1.9 & ref & ref \\
\hline Yes & 3,396 & 27 & $2.73(2.07-3.60)$ & $2.0(1.45-2.63)$ \\
\hline
\end{tabular}

raw fish 2.9(2.44 to 3.34) and (i) eating unripe papaya salad with fermented fish 2.7(2.07 to 3.60). Multiple logistic Regression Analysis was the used to investigate Ophisthorchis infection factors. The remaining 6 variables were: $i$ ) sex; $i$ ) age; $i i i)$ water resource habitat; $i v$ ) having used to take medicine for Ophisthochis infection; $v$ ) having an Ophisthochis infection record and vi) eating raw fish, raw fish salad or fermented fish (Table 3).

\section{Discussion}

We tested the stool of 3,916 subjects for Opisthorchis (using Modified Kato Katz). The respective sensitivity and specificity of this method is $85.5 \%$ and $68.7 \%$ (Stensvold et al., 2006). We discovered that the prevalence of Opisthorchis was $22.7 \%$ while the highest prevalence in one village was $80 \%$-these numbers are comparable to the $0-80 \%$ found in other studies (Sriamporn et al., 2004; Rangsin et al., 2009; Saengsawang et al., 2012). Infected patients under 20 represented $26.2 \%$ of the total. In areas where prevalence was 0 , villagers took medicine a few times a year. Saengsawang et al. (2013) discovered that $60 \%$ of people took praziquantel for opisthorchiasis and yet the prevalence in upper Isaan has not been significantly reduced, which seems paradoxical since overall the infection rate in Thailand as a whole has fallen (Wongsaroj et al., 2009) and the number of liver cancer patients and cholangiocarcinoma patients has been stable (Sriamporn et al., 2004).

The highest prevalence was among those between 50 and 59 years of age (31.5\%) (29.2\% more males than females); similar to the studies by other studied (kaewpitoon et al., 2012a; 2012b). By contrast, Sangsawang et al. (2013) discovered that OV infection was highest in the 20-35 year age group. Men drink alcohol in groups and 'prove' themselves by eating anything, cooked or uncooked. As Rangsin et al (2009). The study revealed that people in 50-60 year were still eating foods at risk of causing infection.

People who lived near canal, lake or river were 1.5 times more at risk of Opisthorchis infection than those who lived several kilometers away; due to disease vectors such as snails, their metacercania and the cyprinoid fish that they infect (Forrer et al., 2012). By contrast, Tesana et al. (1991) discovered that even villages relatively from a river had higher infection (52.2\%) than those nearer (24.7) $(\mathrm{p}<0.005)$. Eating raw freshwater scaly fish (Cyprinids) and fermented fish paste (wherever it is made or by whom) increases the risk for Opisthorchis infection (Rangsin et al., 2009). Northeast people enjoy the flavor of this fish and believe that fluke infestations can be eliminated thereby removing any risk of cancer (Wongba et al., 2011), so they do not change their behavior and repeatedly re-infect themselves. In this study found that having used to take praziquantel drug associated with $\mathrm{OV}$ infection; similar to the studies by Sangsawang et al. (2013).

A solution for infection prevention needs to be quickly investigated especially for middle-aged males. When people engage in less risky behaviors, the need and consumption of anti-anthelminthic treatment will be reduced. A health education campaign needs to be organized in areas adjacent to rivers and lakes. The respective outreach must match the environmental context, the population demographics and cultural sensitivities. Above all, the youth need to be educated to be the new eating habit role models.

\section{Acknowledgements}

Thanks would go to the National Health Security Office Region 8th for financial support and Mr.Supot Kamsa-ard for data analysis.

\section{References}

Charoensuk L, Pinlaor P, Prakobwong S, et al (2011). Curcumin induces a nuclear factor -erythroid 2-related factor 2-driven response against oxidative and nitrative stress after praziquantel treatment in liver fluke-infected hamsters. $J$ Parasitol, 41, 615-26.

Forrer A, Sayasone S, Vounatsou P, et al (2012). Spatial distribution of, and risk factors for Opisthorchis viverrini infection in Southern Lao PDR. PLoS Negl Trop Dis, 6.

Jongsuksuntigul P, Imsomboon T (2003). Opisthorchiasis control in Thailand. Acta Tropical, 88, 229-32.

Kaewpitoon SJ, Rujirakul R, Ueng-arporn N, et al (2012). Community-based cross-sectional study of carcinogenic human liver fluke in elderly from Surin province, Thailand. Asian Pacific J Cancer Prev, 13, 4285-8.

Kaewpitoon SJ, Rujirakul R, Kaewpitoon N (2012). Prevalence of Opisthorchis viverrini infection in Nakhon Ratchasima province, Northeast Thailand. Asian Pacific J Cancer Prev, 13, 5245-9.

Rangsin R, Mungthin M, Taamasri P et al (2009). Incidence and risk factors of Opisthorchis viverrini infections in rural 
Kesorn Thaewnongiew et al

community in Thailand. Am J Trop Med Hyg, 81, 152-5.

Saengsawang P, Promthet S, Bradshaw P (2012). Prevalence of OV infection in Yasothon province, Northeast Thailand. Asian Pac J Cancer Prev, 13, 3399-402.

Saengsawang P, Promthet S, Bradshaw P (2013). Infection with Opisthorchis viverrini and use of praziquantel among a working-age population in Northeast Thailand. Asian Pac J Cancer Prev, 14, 2963-6

Sithithaworn P,Andrews RH, Van De N, et al (2012). The current status of opisthorchiasis and clonorchiasis in the Mekong basin. Parasitology Int, 61, 10-6.

Sriamporn S, Pisani P, Pipitgool V, et al (2004). Prevalence of Opisthorchis viverrini infection and incidence of cholangiocarcinoma in Khon Kaen, Northeast Thailand. Trop Med International Health, 9, 588-94.

Sripa B, Kaewkes S, Intapan PM, Maleewong W, Brindley PJ (2010). Food-borne trematodiasesin Southeast Asia: epidemiology, pathology, clinical manifestation and control. Adv Parasitology, 72, 305-50.

Stensvold CR, Saijuntha W, Sithithaworn P, et al (2006). Evaluation of PCR base coprodiagnosis of human opisthorchiasis. Acta Tropica, 97, 26-30.

Kamsa-ard S, Laopaiboon M,Luvira V, Bhudhisawasdi V (2013). Association between praziquantel and cholangiocarcinoma in patients infected with Opisthorchis viverrini: a systematic review and meta-analysis. Asian Pac J Cancer Prev, 14, 7011-6.

Tesana S, Sithithaworn P, Prasongwatana J ,et al (1991). Influence of water current on the distribution of Opisthorchis viverrini infection in northeastern villages of Thailand. Southeast Asian J Trop Med Hlth, 22, 93-8.

Vatanasapt V, Tangvoraphonkchai V, Titapant V, et al (1990). A high incidence of liver cancer in khon kaen province, Thailand. Southeast Asian J Trop Med Public Health, 21, 489-94.

Wattanayingcharoenchai S, Nithikathkul C, Wongsaroj T, et al (2011). Geographic information system of Opisthorchis viverrini in northeast Thailand. Asian Biomedicine, 687-91.

Wongba N, Thaewnongiew K, Phathee K et al (2011). Liver fluke prevention and control in the Northeast of Thailand through action research. Asian Pac J Cancer Prev, 12, 1-4.

Wongsaroj T, Kairat D, Ramsut P et al (2009). Situation of helminthlasis and protozoa in Thailand, 2009. 